\title{
ACCURACY OF APPROXIMATE METHODS OF UNCERTAINTY PROPAGATION IN SEISMIC LOSS ESTIMATION
}

\author{
Brendon A Bradley ${ }^{1 *}$, Dominic S Lee ${ }^{2}$. \\ ${ }^{1}$ Department of Civil Engineering, University of Canterbury, Private Bag 4800, Christchurch 8020, New Zealand \\ ${ }^{2}$ Department of Mathematics and Statistics, University of Canterbury, Private Bag 4800, Christchurch 8020, New \\ Zealand \\ *Corresponding author: Ph +64-3-366 7001 ext 7673; Fax:+64-3-364 2758; bab54@student.canterbury.ac.nz
}

\begin{abstract}
In this paper the efficacy of an approximate method of uncertainty propagation, known as the first-order second-moment (FOSM) method, for use in seismic loss estimation is investigated. The governing probabilistic equations which define the Pacific Earthquake Engineering Research (PEER)-based loss estimation methodology used are discussed, and the proposed locations to use the FOSM approximations identified. The justification for the use of these approximations is based on a significant reduction in computational time by not requiring direct numerical integration, and the fact that only the first two moments of the distribution are known. Via various examples it is shown that great care should be taken in the use of such approximations, particularly considering the large uncertainties that must be propagated in a seismic loss assessment. Finally, a complete loss assessment of a structure is considered to investigate in detail the location where significant approximation errors are incurred, where caution must be taken in the interpretation of the results, and the computational demand of the various alternatives.
\end{abstract}

\section{KEYWORDS}

Performance-based earthquake engineering (PBEE); aleatory uncertainty; epistemic uncertainty; first-order second-moment (FOSM) method; loss estimation; loss deaggregation. 


\section{INTRODUCTION}

There are many uncertainties in earthquake engineering, ranging from uncertainties in the spatial and temporal occurrence and size distribution of earthquakes, to the uncertainties in the structural response for a given level of ground motion shaking, and the damage and loss consequences as a result of the structural response. In contemporary performance-based earthquake engineering (PBEE), there is a need to account for such uncertainties when characterising the performance of a structural system vulnerable to earthquake hazards. Great progress has been made in frameworks which consistently account for all of the aforementioned uncertainties, primarily since the presentation of the so-called "PEER framing formula" [1,2] advocated by the Pacific Earthquake Engineering Research (PEER) centre. The PEER framing formula allows explicit and consistent treatment of uncertainties in the process of PBEE. The key assumption of conditional independence allows the framing formula to be separated into four different relationships, which are typically solved by different expert personnel, and then integrated together to provide decision variables for stakeholders to make rational decisions regarding seismic risk.

In order to conduct loss assessments within such a framework by combining the aforementioned relationships, the uncertainties in each of the steps must be propagated through to the final decision variables. This uncertainty propagation can be done in various ways with various levels of sophistication and accuracy. The primarily continuous nature of the relationships which comprise the PEER framing formula results in uncertainty propagation via integration over the domain of the random variables. Despite the ever increasing processing abilities of modern computing, as the dimensionality of the integral equations increase, the computational work to perform seismic loss estimation can still be demanding. As a result, there is still a desire by many to develop uncertainty propagation methods which allow results to be obtained efficiently, without significant loss of accuracy. In particular, the 
moment-method approach of approximating probability density functions instead of using approximate (i.e. numerical) integration methods has become popular. For example, secondmoment methods, in which only the first two moments (mean and standard deviation) of the random variables are retained, have been used widely in design code calibration (e.g. [3]).

Regarding uncertainty propagation specifically with the PEER seismic loss estimation framework, use of the first-order second-moment (FOSM) method has been proposed by Baker and Cornell $[4,5]$. The FOSM method uses a first-order Taylor Series approximation of a function of random variables, with each random variable being approximated by its first two moments. Baker and Cornell use the FOSM method primarily for computation of the relationship between a vector of losses and ground motion intensity, which they identify as the computationally demanding step of the seismic loss estimation process. A similar approach has also been taken by Aslani [6], who uses the FOSM method for determination of the covariance structure of the loss given intensity relationship. Despite this use of the FOSM method in seismic loss estimation, as far as the authors are aware, to date its accuracy and efficiency in reducing the computational demand has not yet been scrutinized in detail.

In this manuscript, the use of the FOSM method in various stages of the PEER framework formula is investigated. Its limitations regarding accuracy in the computation of various measures of seismic performance is illustrated through application to the loss assessment of a building, and comparison with the direct solution via numerical integration. The computational demands of the FOSM methods are also discussed in order for the benefits and shortfalls of the method to be properly put in perspective.

\section{PROBABILISTIC SEISMIC LOSS ESTIMATION FRAMEWORK}

For completeness, a review of the PEER loss assessment framework equations is given in this section, which also forms the basis for the FOSM approximations investigated 
throughout the remainder of this paper. In this section we consider aleatory uncertainties only. The inclusion and propagation of epistemic uncertainties is discussed briefly later in the manuscript.

The PEER loss estimation framework is built around the following equation [5]:

$$
\lambda_{L_{T}}(z)=\int_{\mathbf{u}} \int_{\mathbf{v}} \int_{\mathbf{y}} \int_{x} G_{L_{T} \mid \mathbf{L}}(z \mid \mathbf{u}) f_{\mathbf{L} \mid \mathbf{D S}}(\mathbf{u} \mid \mathbf{v}) f_{\mathbf{D S} \mid \mathbf{E D P}}(\mathbf{v} \mid \mathbf{y}) f_{\mathbf{E D P} \mid I M}(\mathbf{y} \mid x)\left|\frac{\mathrm{d} \lambda_{I M}(x)}{\mathrm{d} x}\right| \mathrm{d} \mathbf{u} \mathrm{d} \mathbf{v} \mathrm{d} \mathbf{y} \mathrm{d} x
$$

where $I M$ is an intensity measure representing the intensity of the ground motion observed at the site of the structure; $\boldsymbol{E D P} \mid I M$ is a vector of engineering demand parameters which characterise the response of the structure for a given level of ground motion $I M=i m ; \boldsymbol{D S} \mid \boldsymbol{E D P}$ is a vector of damage states for each component given demand $\boldsymbol{E D P}=\boldsymbol{e d p} ; \boldsymbol{L} \mid \boldsymbol{D S}$ is a vector of losses incurred in each component due to damage states, $\mathbf{D S}=d s ; L_{T}$ is the total loss in the structure; $G_{X \mid Y}(x \mid y)=\mathrm{P}(X \geq x \mid Y=y)$ is the complementary cumulative distribution function $(\mathrm{CCDF})$ of $X$ given $Y=y ; f_{X \mid Y}(x \mid y)$ is the probability density of $X$ given $Y=y$; and $\lambda_{Z}(z)$ is the annual frequency of $Z$ exceeding $z$. Herein for brevity we will use the shorthand notation $G_{X \mid Y}$ , $f_{X \mid Y}$, and $\lambda_{Z}$ in place of $G_{X \mid Y}(x \mid y), f_{X \mid Y}(x \mid y)$, and $\lambda_{Z}(z)$, respectively. Equation (1) represents an application of the total probability theorem. As previously mentioned, Equation (1) also makes the conditional independence assumption (e.g. that the damage state, $\boldsymbol{D S}$, is only a function of $\boldsymbol{E D P}$, and not of the $I M$ causing $\boldsymbol{E D P}$ ), and therefore can be de-coupled and solved in separate stages.

All components which are used to describe the structure and its inventory are defined by fragility and loss functions. Fragility and loss functions can be combined (for a single component) to obtain the relationship between component loss for a given $E D P(L \mid E D P)$ (e.g. [6]). From the results of seismic response analysis a relationship can be found between ground motion intensity measures (IM's) and various EDP's throughout the structure. This $E D P \mid I M$ relationship can be combined with the $L \mid E D P$ relationship to obtain a relation between the loss for a single component, $k$, (which is dependent on $E D P_{i}$ ) given a certain level 
of $\operatorname{IM}\left(L_{k} \mid I M\right)$ :

$$
\begin{aligned}
& \mu_{L_{k} \mid I M}=\int \mu_{L_{k} \mid E D P_{i}} f_{E D P_{i} \mid I M} \mathrm{~d} E D P \\
& \sigma_{L_{k} \mid I M}^{2}=\int\left[\mu_{L_{k} \mid E D P_{i}}^{2}+\sigma_{L_{k} \mid E D P_{i}}^{2}\right] f_{E D P_{i} \mid I M} \mathrm{~d} E D P-\mu_{L_{k} \mid I M}^{2}
\end{aligned}
$$

where $\mu_{L_{k} \mid I M}$ is the mean loss for component $k$ given $I M=i m ; f_{E D P_{i} \mid I M}$ is the probability density function of $E D P_{i}$ given $I M=i m ; \mu_{L_{k} \mid E D P_{i}}$ is the mean loss for component $k$ given $E D P_{i}=e d p_{i}$; and $\sigma_{L_{k} \mid E D P_{i}}^{2}=$ the variance in the loss for component $k$ given $E D P_{i}=e d p_{i}$.

Equations (2) and (3) can be used to obtain the first two moments for the distribution of loss given $I M$ for a single component $k$. For the case where the structure does not collapse it is reasonably assumed that the total direct repair loss for the entire structure can be obtained from the summation of the direct repair losses in each of the different components. More specifically:

$$
\begin{aligned}
\mu_{L_{T} \mid I M, N C} & =\sum_{k=1}^{N c} \mu_{L_{k} \mid I M} \\
\sigma_{L_{T} \mid I M, N C}^{2} & =\sum_{k=1}^{N c} \sigma_{L_{k} \mid I M}^{2}+2 \sum_{k=1}^{N c} \sum_{k^{\prime}=1}^{k-1} \sigma_{L_{k}\left|I M, L_{k^{\prime}}\right| I M}
\end{aligned}
$$

where $N_{c}$ is the number of components in the structure; and $\sigma_{L_{k}\left|I M, L_{k^{\prime}}\right| I M}$ is the covariance in the $L \mid I M$ relationship between components $k$ and $k$ '. This covariance can be computed by:

$$
\sigma_{L_{k}\left|I M, L_{k^{\prime}}\right| I M}=\mu_{L_{k} L_{k^{\prime}} \mid I M}-\mu_{L_{k} \mid I M} \mu_{L_{k^{\prime}} \mid I M}
$$

In which $\mu_{L_{k} L_{k^{\prime}} \mid I M}$ is computed from:

$$
\mu_{L_{k} L_{k^{\prime}} \mid I M}=\iint\left(\mu_{L_{k} \mid E D P_{i}} \mu_{L_{k^{\prime}} \mid E D P_{i^{\prime}}}+\sigma_{L_{k}\left|E D P_{i}, L_{k^{\prime}}\right| E D P_{i^{\prime}}}\right) f_{E D P_{i}\left|I M, E D P_{i^{\prime}}\right| I M} \mathrm{dE} D P_{i} \mathrm{dE} D P_{i^{\prime}}
$$

where $f_{E D P_{i}\left|I M, E D P_{i}\right| I M}$ is the joint distribution of $E D P_{i}$ and $E D P_{i}$, given $I M=i m$; and $\sigma_{L_{k}\left|E D P_{i}, L_{k^{\prime}}\right| E D P_{i^{\prime}}}$ is the covariance in the $L \mid E D P$ relationship for components $k$ and $k^{\prime}$.

By considering the mutually exclusive and collective exhaustive events of collapse and 
no collapse, the expected loss for a given $I M$ can be computed from:

$\mu_{L_{T} \mid I M}=\mu_{L_{T} \mid I M, N C}\left\lfloor 1-P_{C \mid I M}\right\rfloor+\mu_{L_{T} \mid C} P_{C \mid I M}$

where $\mu_{L_{T} \mid C}$ is the mean of the loss given global collapse (assumed independent of $\left.I M\right) ; \mu_{L_{T} \mid M}$ is the mean of the total loss once conditioning on collapse (and no collapse) has been removed; and $P_{C \mid I M}$ is the probability of collapse given $I M$. The standard deviation of the total loss given $I M, \sigma_{L_{T} \mid M M}^{2}$, although not shown here can be computed in a similar manner (See [5, 7] for details).

The expected annual loss can be computed by integrating the expected loss for a given level of ground motion intensity, $\mu_{L_{T} \mid I M}$, with the ground motion hazard curve, $\lambda_{I M}$, which gives the annual frequency of exceeding different levels of ground motion intensity.

$\mu_{L_{T}}=\int \mu_{L_{T} \mid I M}\left|\frac{d \lambda_{I M}}{d I M}\right| d I M$

The loss hazard curve gives the annual frequency of exceeding a specified value of loss, and can be computed from:

$\lambda_{L_{T}}=\int G_{L_{T} \mid I M}\left|\frac{d \lambda_{I M}}{d I M}\right| d I M$

where $G_{L_{T} \mid I M}$ is the CCDF of the total loss given $I M$.

\section{APPROXIMATE UNCERTAINTY PROPAGATION}

It is proposed herein to investigate the efficacy of using the FOSM method in place of some of the equations in the integral-based loss estimation methodology presented in the previous section. Initially, the same stance as that of Baker and Cornell [5] is taken for the location of the FOSM approximations. That is, the FOSM method is used primarily in computing the moments of the $L \mid I M$ relationship since in most practical situations there will be many different components and therefore this step of the loss estimation methodology is 
potentially computationally intensive. Direct numerical integration is used for equations with the ground motion hazard (i.e. Equations (9) and (10)), since the ground motion hazard is a dominant contributor toward the total uncertainty, and also the dimensionality of the integral is one (hence the computational demand is relatively small in comparison to the computation of the moments of the $L \mid I M$ relation). The following section gives an overview of the development of the FOSM method and its limitations to some general problems; its use for the particular seismic loss assessment problem is discussed in subsequent sections.

\section{First order second moment (FOSM) method}

Consider a general scalar function $Y=\mathrm{g}(X)$ where $X$ is a random variable. The moments (mean, variance, etc) of $Y$ can be obtained directly by integrating the product of $g(X)$ and $f_{\mathrm{X}}(x)$ over the support of $X[8]$ (the particular form of the product depends on the moment being computed), where $f_{\mathrm{X}}(x)$ is the probability density function of $X$. Under certain conditions, it may be appropriate to approximate these moments based on a Taylor series expansion of $g(X)$ about the mean value of $x$, that is:

$$
g(X)=g\left(\mu_{X}\right)+\left(X-\mu_{X}\right) \frac{d g}{d X}+\frac{1}{2 !}\left(X-\mu_{X}\right)^{2} \frac{d^{2} g}{d X^{2}}+\ldots
$$

where all of the derivatives are evaluated at $X=\mu_{X}$.

From Equation (11) and using the fact that $E[Y]=E[g(X)]$ and $\operatorname{Var}[Y]=E\left[g(X)^{2}\right]$ $E[g(X)]^{2}$, a first order estimate of the mean and variance of $Y$ are:

$$
\begin{aligned}
& E[Y]=\mu_{Y} \approx g\left(\mu_{X}\right) \\
& \operatorname{Var}[Y]=\left.\sigma_{Y}^{2} \approx \sigma_{g(X)}^{2}\right|_{X=\mu_{X}}+\left.\sigma_{X}^{2}\left(\frac{d g}{d X}\right)^{2}\right|_{X=\mu_{X}}
\end{aligned}
$$

where $\sigma_{g(X)}^{2}$ is the variance in $g(X)$ and $\sigma_{X}^{2}$ is the variance in $X$ itself, both of which are

evaluated at $X=\mu_{X}$. The derivative, $\frac{d g}{d X}$, can be thought of as the sensitivity of changes in $X$ to the resulting $Y$. Equations (12) and (13) define what is known as the first-order-second 
moment (FOSM) method in structural reliability [9]. The FOSM method is useful as the first two moments of the function $Y=g(X)$ can be obtained without the need for integration, and only the first two moments of $X$ are required. In some instances the second order estimate of the mean can also be used (which still requires only the first two moments of $X$ ). The expectation of the second order truncation of Equation (12) yields:

$$
E[Y]=\mu_{Y} \approx g\left(\mu_{X}\right)+\left.\frac{1}{2} \sigma_{X}^{2} \frac{d^{2} g}{d X^{2}}\right|_{X=\mu_{X}}
$$

Thus, unlike the first order estimate of the mean (Equation (12)) which is independent of the variance of $X$, the second order estimate of the mean (Equation (14)) accounts for the variance in $X$, which also depends on the second derivative (curvature) of $g(X)$. Below, two problems are considered to illustrate some salient features of the approximate methods given by Equations (12)-(14).

\section{Example 1: $\mathrm{Y}=\mathrm{X}^{2}$.}

In this first example consider the case $Y=g(X)=X^{2}$. It is assumed that $X$ has a lognormal distribution with a mean, $\mu_{X}=2$ and coefficient of variation $(\mathrm{COV})$, $\delta_{X}=\sigma_{X} / \mu_{X}$ varying from 0.01 to 1.0 . As $g(X)$ is quadratic (and thus its second derivative is constant) then the second order estimate of the mean (Equation (14)) is exact and forms the basis for the accuracy of the FOSM method in this case. The exact and FOSM approximations for the moments of $Y$ are:

$$
\begin{aligned}
& \mu_{Y}=\mu_{X}{ }^{2}+\sigma_{X}{ }^{2} \approx \mu_{X}{ }^{2} \\
& \sigma_{Y}^{2}=\left(\mu_{X}^{2}+\sigma_{X}^{2}\right)^{2}\left[\left(\frac{\sigma_{X}^{2}}{\mu_{X}^{2}}+1\right)^{4}-1\right] \approx 4 \sigma_{X}^{2} \mu_{X}^{2}
\end{aligned}
$$

Figure 1 illustrates the corresponding errors in the first order mean and standard deviation estimations of $Y=X^{2}$. It is observed that the first order estimate of the mean always under predicts the value of $\mu_{Y}$ (as is obvious from Equation (15)), with the relative error 
increasing as the COV increases. The error in the standard deviation also increases as the $\mathrm{COV}$ increases, and in particular, for a given $\mathrm{COV}$ the error in the standard deviation is notably higher than for the mean approximation. For example, for a $\mathrm{COV}$ of 0.6 the relative error in the mean is approximately $-26 \%$, while the error in the standard deviation is approximately $-77 \%$. These correspond to error ratios (i.e. the exact value divided by the FOSM value) of 1.35 and 3.1 , respectively.

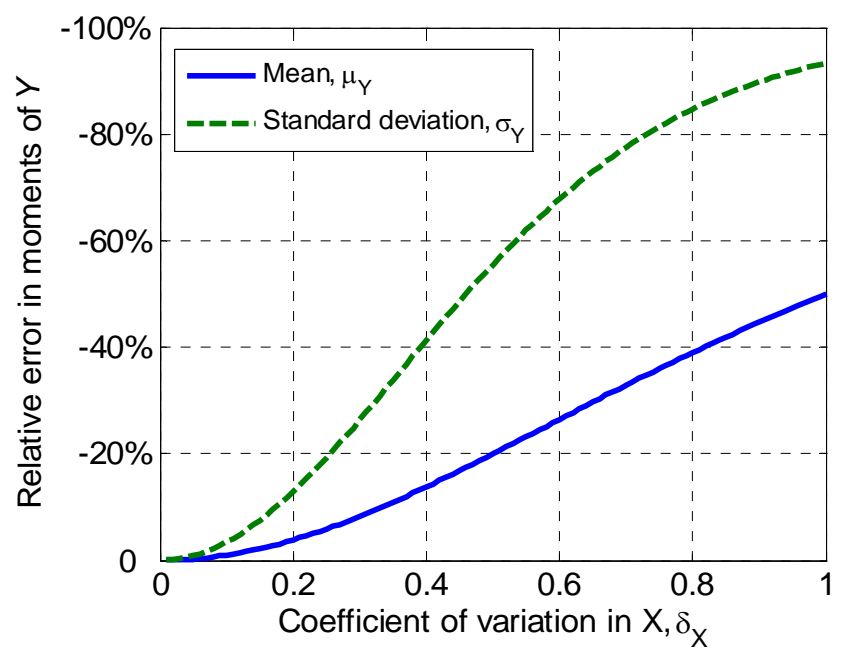

Figure 1: Errors in the mean and standard approximation for Example 1.

\section{Example 2: Second-order mean approximation.}

In the second example consider the use of both the first- and second-order methods for estimation of the expectation of $Y=\mathrm{g}(X)$ for various levels of uncertainty in $X$. As the particular results may be dependent on the analytical form of $g(X)$, relationships are used which represent the general trends in the relationships between loss and demand $(L \mid E D P)$, and between demand and intensity $(E D P \mid I M)$ for the seismic loss estimation problem. The (normalised) expected $L \mid E D P$ relationship is based on a component with a single damage state having a lognormal fragility curve with mean, $\mu_{\ln E D P \mid D S}=-1.20$ (equivalent to a median of $\overline{E D P}=0.3$ ) and lognormal standard deviation (dispersion), $\sigma_{\ln E D P \mid D S}=0.3$. The expected $E D P \mid I M$ relationship used is of a power model form, $\mu_{E D P \mid I M}=0.0025 I M^{2}$, which has been used by numerous researchers.

Figure 2 illustrates the accuracy of the first- and second-order approximations for 
various dispersion values in the $E D P \mid I M$ relationship. Figure 2a illustrates that for small values of the dispersion $\left(\sigma_{\operatorname{lnEDPIIM}}=0.15\right)$ the second-order approximation is significantly better than the first-order approximation, effectively lying over the exact curve obtained via numerical integration. For a moderate value of dispersion $\left(\sigma_{\ln E D P \mid I M}=0.25\right)$, the accuracy of the second-order approximation is good for $I M$ values which correspond to loss values below 0.5 , but for loss values above 0.5 , there is some deviation of the second-order approximation from the exact solution for larger $I M$ values. Note that the first order approximation (Equation (12)) is independent of the uncertainty in the $E D P \mid I M$ relationship, thus yielding the same result for all dispersion values.
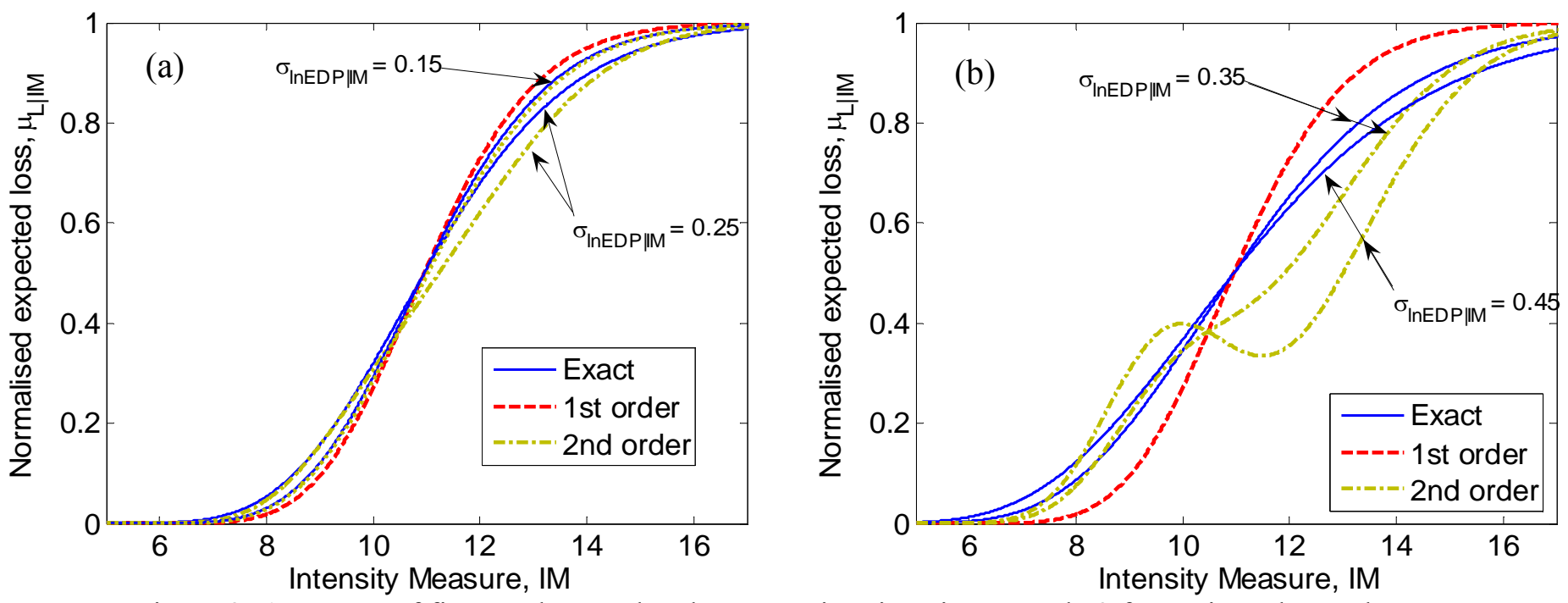

Figure 2: Accuracy of first- and second-order approximations in Example 2 for various demandintensity dispersions, $\sigma_{\ln E D P \mid I M}$.

Figure $2 \mathrm{~b}$ illustrates the accuracy of the approximations for larger values of the dispersion in the $E D P \mid I M$ relationship. In this case we can see that in the vicinity of $\mu_{L \mid I M}=0.5$ there is significant error in the second-order approximation, which increases with increasing dispersion. Careful inspection of Equation (14) and the second derivative of the $L \mid E D P$ relationship illustrates that this error is caused by the second derivative of the $L \mid E D P$ relationship, which is largest either side of $\mu_{L \mid I M}=0.5$. As it is by no means uncommon to have uncertainties this large in the $E D P \mid I M$ relationship, then primarily because of this 
apparent 'instability' of the second-order method, we will use the first-order approximation of the mean throughout the remainder of this manuscript.

\section{APPROXIMATE SEISMIC LOSS ASSESSMENT}

In this section consideration is given to the use of the FOSM method within the seismic loss assessment framework. As it is common within the loss assessment framework to assume that the distribution of the demand given intensity relationship $(E D P \mid I M)$ is lognormal then log-forms are used within the FOSM method (such that the $\ln E D P \mid I M$ distribution is normal) and then are later converted back to non-log forms.

\section{Expected loss given intensity measure, $E[L \mid I M]$}

The FOSM approximation for the expected loss given $I M$ for component $k$ (computed directly using Equation (2)) can be shown to equal [5]:

$$
\mu_{\ln L_{k} \mid I M} \cong \mu_{\ln L_{k} \mid \ln E D P_{i}}\left(\mu_{\ln E D P_{i} \mid I M}\right)
$$

That is, $\mu_{\ln L_{k} \mid I M}$ is obtained by computing $\mu_{\ln L \mid \ln E D P_{i}}$ at the mean value of $\ln E D P_{i} \mid I M$, $\mu_{\ln E D P_{i} \mid I M}$. The non-log form is then obtained by the first-order approximation $\mu_{L_{k} \mid M M} \cong e^{\mu_{\ln L_{k} \mid M M}}$

To investigate the accuracy of the FOSM approximation for $\mu_{L_{k} \mid I M}$, two different types of components are selected. The first is a reinforced concrete (RC) column and the second an interior partition. The properties of the damage state fragility and loss functions are given in Table 1. In Table 1 , the EDP of peak interstorey drift $\left(\theta_{\max }\right)$ values have been obtained from the deformation damage index (DDI) values given in Mitrani-Reiser [10] using recoverable elastic and ultimate rotations of $\theta_{r}=0.005$ and $\theta_{u}=0.06$, respectively. The repair costs for the $\mathrm{RC}$ column are per column costs, while for the interior partition the costs are per $64 \mathrm{ft}^{2}[10]$. These two different components have been selected since the RC column has fragility curves 
with large uncertainty $\left(\sigma_{\ln E D P \mid D S}=0.74-1.36\right)$, while the uncertainty in the partition fragility curves is approximately $25 \%$ of those of the column $\left(\sigma_{\ln E D P \mid D S}=0.17,0.23\right)$. The uncertainty in the fragility curves directly affects the second and higher order derivatives of the loss given demand relationship, and it will be shown that this affects the error in the FOSM approximation.

Table 1: Damage state and loss properties for the two components considered

\begin{tabular}{|c|c|c|c|c|c|c|}
\hline & \multicolumn{3}{|c|}{ RC column $^{1,2}[10]$} & \multicolumn{2}{c|}{ Internal Partition $^{1}[10]$} \\
\hline & $D S_{1}$ & $D S_{2}$ & $D S_{3}$ & $D S_{4}$ & $D S_{1}$ & $D S_{2}$ \\
\hline$\mu_{E D P \mid D S}$ & 0.0044 & 0.017 & 0.039 & 0.070 & 0.0039 & 0.0085 \\
\hline$\sigma_{\ln E D P \mid D S}$ & 1.36 & 0.89 & 0.80 & 0.74 & 0.17 & 0.23 \\
\hline$\mu_{L \mid D S}$ & 8.0 & 22.5 & 34.3 & 34.3 & 0.088 & 0.525 \\
\hline$\sigma_{\ln L \mid D S}$ & 0.42 & 0.40 & 0.37 & 0.37 & 0.2 & 0.2 \\
\hline
\end{tabular}

${ }^{1} E D P$ is max interstory drift, and loss is in thousand USD.

${ }^{2}$ DDI values from Mitrani-Reiser [10] converted to $\theta_{\max }$ using $\theta_{r}=0.005$ and $\theta_{u}=0.06$.

Figure $3 \mathrm{a}$ and Figure $3 \mathrm{~b}$ illustrate the results for the expected loss given $I M$ for the column due to $D S_{3}$ occurrence, for dispersions of 0.3 and 0.5 (which are assumed independent of $I M)$ in the $E D P \mid I M$ relationship. The mean $E D P \mid I M$ relationship used corresponds to that of the first floor of the case study structure discussed in the final section of this manuscript. It can be seen that as expected the error is directly related to the magnitude of $\sigma_{\ln E D P \mid I M}$, with a relatively small error when $\sigma_{\ln E D P \mid I M}=0.3$ compared to the error when $\sigma_{\ln E D P \mid I M}=0.5$. As in the previous section it is again noted that the FOSM method underestimates the 'exact' loss for small $I M$ values and over estimates for large $I M$ values. Figure $3 \mathrm{c}$ and Figure $3 \mathrm{~d}$ illustrate the $L \mid I M$ curves for the partition due to the onset of $D S_{2}$. Similar trends are seen with that of the column case, in regard to the error being proportional to $\sigma_{\ln E D P \mid I M}$, and also systematic underestimation and overestimation of the error for small and large $I M$ values, respectively. It is also apparent that the error in the FOSM approximation for the partition is much larger than for the column component. Recall that the accuracy of the approximate methods depends not 
only on the uncertainty in $E D P \mid I M$ relationship (which is the same for both the RC column and the interior partition), but also 'shape' (specifically the derivatives) of the $L \mid E D P$ relationship (i.e. the second-order approximation (Equation (14)) indicates the mean depends on the second derivative of $L \mid E D P$ ). Thus, the smaller uncertainty in the partition component damage state, results in a $L \mid E D P$ relationship which has significantly larger higher order derivatives around the $I M$ causing a normalised loss of 0.5 , relative to the $\mathrm{RC}$ column, and thus why the error is larger for the interior partition.
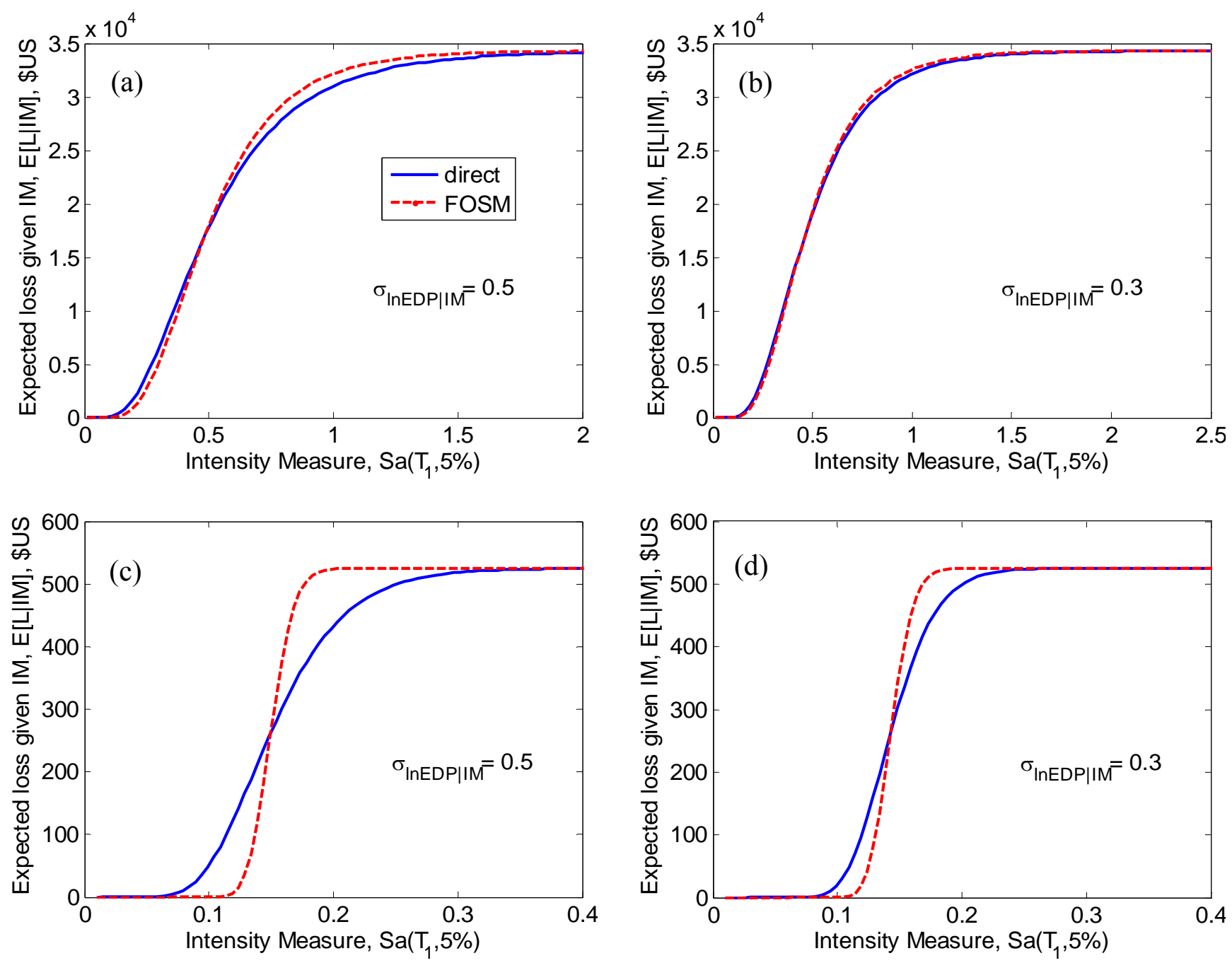

Figure 3: Accuracy of FOSM method for expected loss given IM computation for: (a)\&(b) occurrence of $D S_{3}$ in the RC column component; and (c)\&(d) occurrence of $D S_{2}$ in the partition component.

\section{Standard deviation in loss given intensity measure, $\sigma[L \mid I M]$}

Similar to the mean loss given intensity measure, the standard deviation in the loss given $I M$ can be obtained using a FOSM approximation, which is given by [5]: 


$$
\left.\sigma_{\ln L_{k} \mid I M}^{2} \cong \sigma_{\ln E D P_{i} \mid I M}^{2}\left(\frac{\partial \ln L_{k} \mid \ln E D P_{i}}{\partial \ln E D P_{i}}\right)^{2}\right|_{\mu_{\ln E D P_{i} \mid M}}+\left.\sigma_{\ln L_{k} \mid \ln E D P_{i}}^{2}\right|_{\mu_{\ln E D P_{i} \mid M M}}
$$

Figure $4 \mathrm{a}$ and Figure $4 \mathrm{~b}$ illustrate the dispersion, $\sigma_{\ln L_{k} \mid I M}$, in the $L \mid I M$ relationship for the column component due to the onset of $D S_{3}$ (i.e. analogous to the results in Figure $3 \mathrm{a}$ and Figure $3 \mathrm{~b}$ for the expectation). Again, as for the mean $L \mid I M$ case, it is evident that the error between the FOSM approximation and the direct method is a function of the dispersion in the $E D P \mid I M$ relationship, $\sigma_{\ln E D P \mid I M}$. In both cases, it is also apparent that the FOSM approximation of $\sigma_{\ln L_{k} \mid I M}$ is larger than that obtained by direct integration. The dispersion must next be converted back to the (non-log) standard deviation, which can be achieved in several ways. Firstly, one can make the assumption that the $L \mid I M$ relation has a lognormal distribution (this is the assumption made to get the dispersion from the computed standard deviation in the case of direct integration). Based on this assumption the standard deviation of $L \mid I M$ can be obtained from [8]:

$$
\sigma_{L_{k} \mid I M}=\mu_{L_{k} \mid I M} \sqrt{e^{\sigma_{\ln L_{k} \mid I M}^{2}}-1}
$$

The second approach is to use a first-order approximation [5] to obtain:

$$
\sigma_{L_{k} \mid I M} \cong \mu_{L_{k} \mid I M} \sigma_{\ln L_{k} \mid I M}
$$

Note that Equation (20) can be obtained from Equation (19) by using the Taylor Series approximation: $e^{\sigma^{2}}=1+\sigma^{2}+O\left(\sigma^{4}\right)$.

Figure $4 \mathrm{c}$ and Figure $4 \mathrm{~d}$ illustrate the comparison between the FOSM and direct approaches for computing $\sigma_{L_{k} \mid I M}$, using both Equations (19) and (20) above. It is evident that the first-order assumption (Equation (20)) causes an under approximation in $\sigma_{L_{k} \mid I M}$ even when the dispersion is well predicted (i.e. Figure $4 \mathrm{a}$ and Figure $4 \mathrm{~b}$ ). This occurs because the magnitude of the dispersion, $\sigma_{\ln L_{k} \mid I M}$, is large enough such that the higher order terms in the 
TS approximation for $e^{\sigma_{\ln L_{k} \mid M M}^{2}}$ are significant, particularly at small $I M$ values where $\sigma_{\ln L_{k} \mid I M}$ is well above 1.0 (Figure $4 \mathrm{a}$ and Figure $4 \mathrm{~b}$ ). Using the lognormal assumption to compute $\sigma_{L_{k} \mid I M}$ results in an accurate prediction at large $I M$ levels (i.e. as $\sigma_{L_{k} \mid I M}$ approaches a constant value), however, for smaller $I M$ values it significantly over-predicts $\sigma_{L_{k} \mid I M}$ compared with the direct results. This over-prediction occurs because of the over prediction of $\sigma_{\ln L_{k} \mid I M}$ as shown in Figure 4a and Figure $4 \mathrm{~b}$, which is then amplified through the $e^{\sigma_{\ln L_{k} \mid M M}^{2}}$ term in Equation (19).
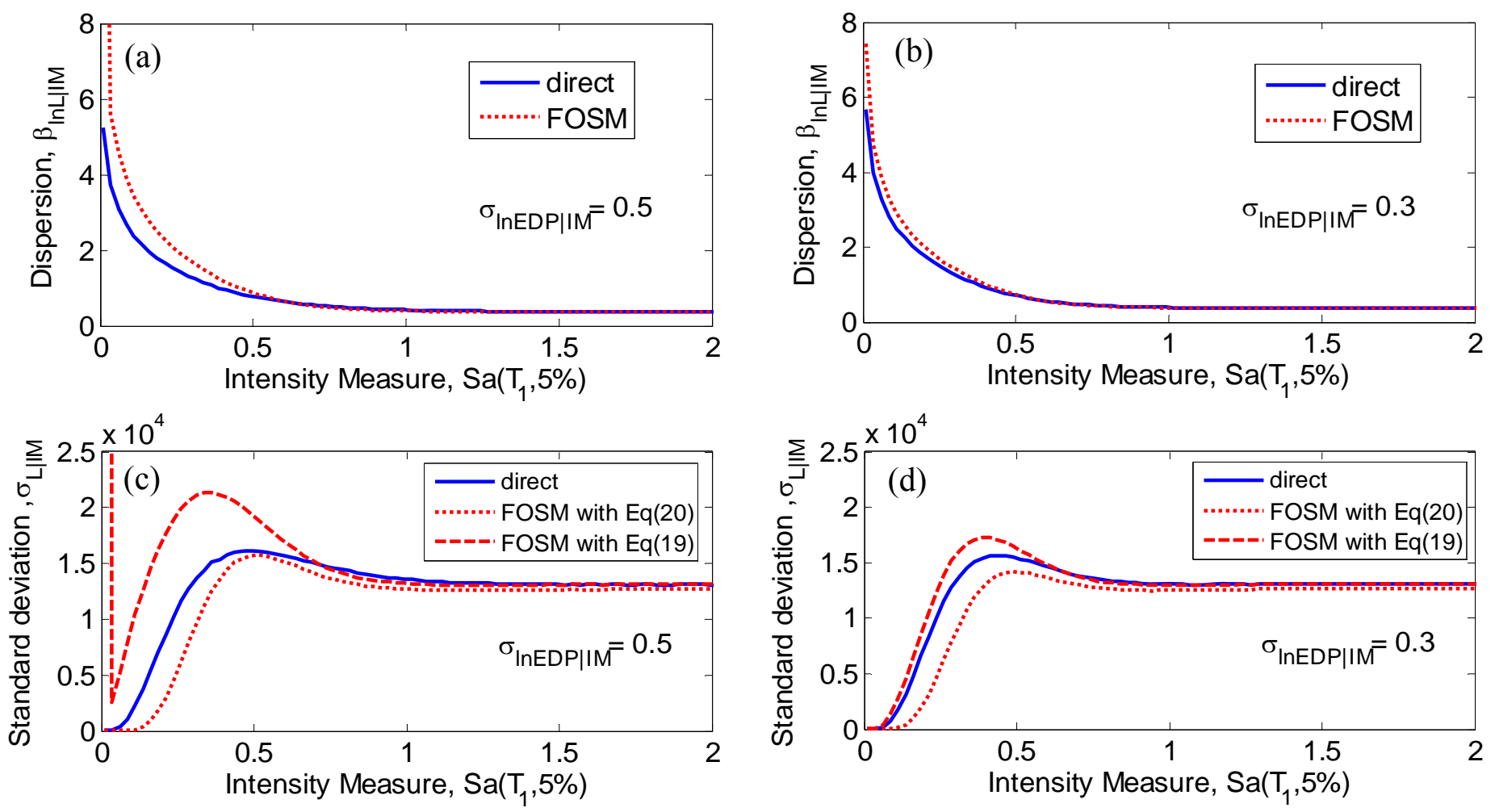

Figure 4: Accuracy of the FOSM method for (a)\&(b) dispersion; and (c)\&(d) standard deviation in the prediction of the uncertainty in the loss given intensity relationship for the column component at two different levels of $E D P \mid I M$ uncertainty.

In the case of Figure 4c, the dispersion using the FOSM method at small IM values is so large that using Equation (19) gives a 'non-convergent' value for $\sigma_{L_{k} \mid I M}$ (i.e. the value of $\sigma_{L_{k} \mid I M}$ does not converge to zero as $\mu_{L_{k} \mid I M}$ tends to zero). This does not occur in Figure $4 \mathrm{~d}$ since the value of $\sigma_{\ln E D P \mid I M}$ is smaller than for Figure $4 \mathrm{c}$, meaning the error in the 
approximation of the dispersion is not as significant. For this reason of 'non-convergence' in $\sigma_{L_{k} \mid I M}$ using the lognormal assumption (Equation (19)), the first-order assumption (Equation (20)) will be used in the remainder of this manuscript.

Figure 5 illustrates the FOSM approximations for the standard deviation in the $L \mid I M$ relationship for the partition component due to the onset of $D S_{2}$ (i.e. analogous to the results in Figure 3 for the expectation). Similar to the expected $L \mid I M$ curves investigated previously it is evident that the approximation is worse for the partition component than the column component due to the small uncertainty in the partition fragility curves. Unlike the column component however, it is observed that around $I M=0.15$ the standard deviation predicted using the FOSM method is quite significantly larger than that obtained via direct numerical integration. It is to be noted that $I M=0.15$ is where the mean loss, $\mu_{L \mid I M}$, is $50 \%$ of its absolute value (e.g. see Figure 3c and Figure 3d). Careful inspection of the standard deviation in loss given intensity for the column component (Figure 4c and Figure 4d) illustrates that there is also 'localised peak' around $I M=0.5$, which is where the mean loss is $50 \%$ of its absolute value (see Figure $3 \mathrm{a}$ and Figure $3 \mathrm{~b}$ ). Therefore this localised peak is evidently occurring about the $I M$ which causes the median $E D P$ of the damage state in the component.
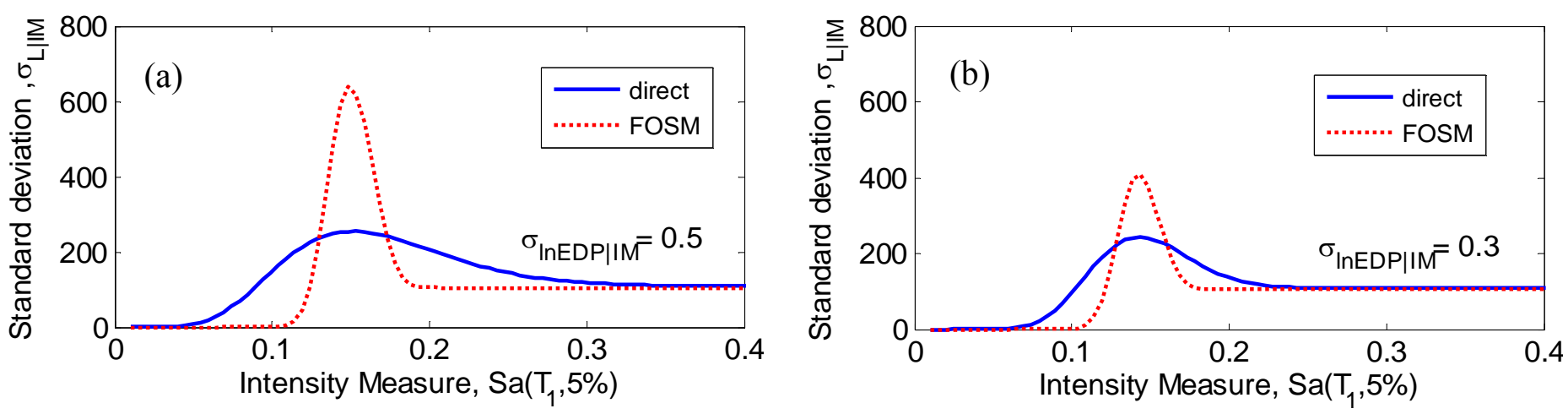

Figure 5: Accuracy of the FOSM prediction for the standard deviation in the loss given intensity relationship for the partition component for: (a) $\sigma_{\ln E D P \mid I M}=0.5$; and (b) $\sigma_{\ln E D P I I M}=0.3$.

\section{Covariance in loss given intensity measure, $\sigma\left[L_{1}, L_{2} \mid I M\right]$}

Equation (5) illustrates that in order to compute the standard deviation in the total loss, it is necessary to compute the covariance in the losses between different components for a 
given level of $I M$. As illustrated by Equation (7) this covariance computation requires knowledge of correlations within the $L|D S, D S| E D P$, and $E D P \mid I M$ relationships. Such correlations occur for a variety of reasons, such as the similar repair actions required between similar components (affects $L \mid D S$ correlation); components made of similar materials (affects $D S \mid E D P$ correlation), and components located in close proximity within the structure (affects $E D P \mid I M$ correlation). As Equation (7) involves double integration it will be shown later that estimation of the covariance is computationally intensive. A FOSM approximation for the covariance of the loss between two components for a given intensity can be shown to be equal to $[5]$ :

$$
\begin{array}{r}
\left.\sigma_{\ln L_{k}\left|I M, \ln L_{k^{\prime}}\right| I M}^{2} \cong \sigma_{\ln E D P_{i}\left|I M, \ln E D P_{i^{\prime}}\right| I M}^{2}\left(\frac{\partial \ln L_{k} \mid \ln E D P_{i}}{\partial \ln E D P_{i}}\right)^{2}\left(\frac{\partial \ln L_{k^{\prime}} \mid \ln E D P_{i^{\prime}}}{\partial \ln E D P_{i^{\prime}}}\right)^{2}\right|_{\mu_{\ln E D P_{i} \mid M M}, \mu_{\ln E D P_{i} \mid I M}} \\
+\left.\sigma_{\ln L_{k}\left|\ln E D P_{i}, \ln L_{k^{\prime}}\right| \ln E D P_{i^{\prime}} \mid}\right|_{\mu_{\ln E D P_{i} \mid M}, \mu_{\ln E D P_{i}|| M}}
\end{array}
$$

where all terms (derivatives and variances) are computed at $\mu_{\ln E D P_{i} \mid I M}$ and $\mu_{\ln E D P_{i} \mid I M}$. The variance given by Equation (21) can then be converted to its non-log form by:

$$
\sigma_{L_{k}\left|I M, L_{k^{\prime}}\right| I M}^{2} \cong \mu_{\ln L_{k} \mid I M} \mu_{\ln L_{k^{\prime}} \mid I M} \sigma_{\ln L_{k}\left|I M, \ln L_{k^{\prime}}\right| I M}^{2}
$$

Due to space limitations, a direct comparison of the FOSM and direct computations of the covariance between two components is not given here. The covariance of the loss between two components is however directly related to the standard deviation in the loss of each of the components and their correlation. As generally the FOSM method leads to an under prediction of the standard deviation in loss given intensity (the exception being for components with small uncertainty in the $D S$ fragility, in which an over approximation occurs about the $I M$ causing the median $E D P$ of the fragility function) it can be inductively stated that the covariance will also likely be under predicted (as positive correlations occur in general).

One further point regarding the computation of the covariance in the loss between two 
components is also warranted. As previously mentioned, the computation of the correlation between losses in different components is a difficult task with a vast lack of data. As such, one may wish to consider upper and lower bounds, by considering no correlations and perfect correlations between components. In the case of no correlations, all covariance terms will vanish and the variance of the total loss will simply be the sum of the variances of each of the components. In the case of assuming perfect correlations between $L|D S, D S| E D P$, and $E D P \mid I M$ relationships for two components that are identical (i.e. means and variances for each of the relationships are equal), the covariance computation (Equations (6) and (7)) will reduce to the product of the standard deviations in the loss for each of the components (Equation (3)). This result is, in general, not true for two different components which have perfect correlations within the $L|D S, D S| E D P$, and $E D P \mid I M$ relationships. That is, in general, even if perfect correlations exist (for two different components) between the three deaggregated $(L|D S, D S| E D P$, and $E D P \mid I M)$ relations the correlation between the $L \mid I M$ relations will not necessarily be one. This point is illustrated for the case study considered later in the manuscript.

\section{Epistemic variance in collapse fragility curve, $\sigma^{2}[\mathrm{P}(C \mid I M)]$}

The FOSM method can also be used when computing the epistemic uncertainties in a cumulative distribution, due to uncertainty in its mean value. In loss estimation methodologies, such a case arises when computing the uncertainty in: (i) the cumulative probability of collapse given IM (for computing the collapse hazard); (ii) the probability of exceeding a given level of demand for a given $I M$ (for computing the demand hazard); and (iii) the probability of exceeding a given level of loss for a given IM (for computing the loss hazard). The demand and loss hazard cases are further complicated in that the cumulative probability distribution needs to also account for the probability of collapse. Details on the methodological formulation to include collapse can be found in Baker and Cornell [4]. As the 
cumulative distribution (which we will assume to be lognormal [11]) for the collapse probability has less of the aforementioned complications we will use it here to illustrate the accuracy of the FOSM method.

Using Bayes' Theorem [8], it is possible to compute the mean and variance in the probability of collapse given $I M$ due to uncertainty in the mean $I M$ causing collapse from:

$$
\begin{aligned}
& \mu_{P(C \mid I M)}=\int P\left(C \mid I M, \mu_{I M \mid C}\right) f\left(\mu_{I M \mid C}\right) d \mu_{I M \mid C} \\
& \sigma_{P(C \mid I M)}^{2}=\int\left[P\left(C \mid I M, \mu_{I M \mid C}\right)\right]^{2} f\left(\mu_{I M \mid C}\right) d \mu_{I M \mid C}-\mu_{P(C \mid I M)}^{2}
\end{aligned}
$$

where $C$ denotes 'collapse'; and $\mu_{I M \mid C}$ is the mean $I M$ causing collapse which is an uncertain quantity. For the particular case of the probability of collapse being a lognormal random variable then FOSM approximation for Equation (24) is [5]:

$$
\sigma_{P(C \mid I M)}^{2} \cong \phi\left(\frac{\ln (i m)-\bar{\mu}_{\ln I M \mid C}}{\sigma_{R}}\right)^{2} \frac{\sigma_{\mu_{\ln I M \mid C}}^{2}}{\sigma_{R}^{2}}
$$

where $\sigma_{R}$ is the aleatory uncertainty in the collapse fragility curve; $\sigma_{\mu_{\mathrm{n} I M C}}$ is the epistemic uncertainty in the (logarithmic) mean $I M$ causing collapse, $\mu_{\ln I M \mid C}$; and $\bar{\mu}_{\ln I M \mid C}$ denotes the mean (with respect to epistemic uncertainty) estimate of $\mu_{\ln I M \mid C}$.

Figure 6a illustrates the accuracy of the FOSM method for relatively typical values of the aleatory and epistemic uncertainties (actual values are usually in the range 0.4-0.5 [12]). It is observed that for $I M$ values near the mean $I M$ causing collapse the FOSM approximation is too large relative to the direct computation, while for $I M$ values at several standard deviations from the mean the FOSM approximation actually falls below the direct values. Figure $6 \mathrm{~b}$ illustrates a slightly more unusual scenario with large aleatory and small epistemic uncertainties. Despite the small value of the epistemic uncertainty in this case, the prediction of the FOSM approximation of the direct solution obtained via numerical integration is poor (note however that the absolute value of the uncertainty in Figure $6 \mathrm{~b}$ is small relative to 
Figure 6a).
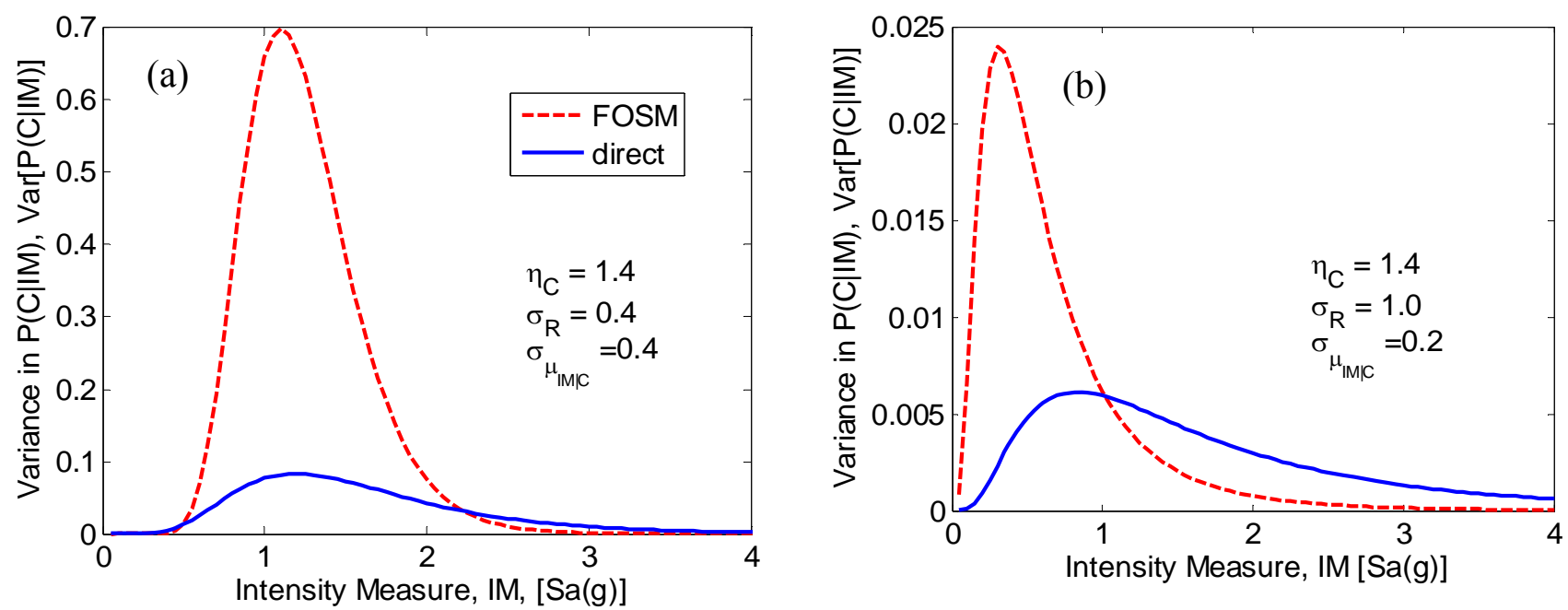

Figure 6: Error in the prediction of the epistemic variance in the cumulative distribution for collapse probability using the FOSM method using: (a) $\eta_{C}=1.4 ; \sigma_{\mathrm{R}}=0.4 ; \sigma_{\mathrm{IIM} \mid \mathrm{C}}=0.4$; and (b) $\eta_{C}=1.4 ; \sigma_{\mathrm{R}}=1.0$; $\sigma_{\mu \mathrm{IM} \mid \mathrm{C}}=0.2$. Here $\eta_{C}$ is the median $I M$ value causing structural collapse.

\section{APPLICATION TO LOSS ASSESSMENT OF A STRUCTURE}

In this section the FOSM method is applied in the loss assessment of a 10 storey reinforced concrete moment frame structure, and compared to the results obtained via direct integration of the loss assessment governing equations. A detailed discussion on the case study structure and its loss assessment is given in [7], and briefly explained below.

\section{Case study structure}

The case study structure used herein to illustrate the accuracy of the FOSM method for the loss estimation of an entire structure is based on the Red Book building [13] which acts as a design example of the New Zealand Concrete Code [14]. The primary lateral load carrying system consists of four one-way perimeter moment resisting frames which are 3 bays long. Vertical loads are transferred primarily through interior columns with gravity beams supporting one-way floor units. The loss assessment considers 115 different component groups which encompass structural components, non-structural components and contents. Only losses due to direct repair cost of damage were considered (i.e. human casualties and business disruption were not considered), and epistemic uncertainties were not considered. 


\section{Approximate methods of uncertainty propagation used in loss assessment}

As has been previously discussed, there are several different options for the inclusion of the FOSM method in the seismic loss assessment computations. Here we will compare two different alternatives, which have been used by previous researchers (but not compared against the exact solution).

Baker and Cornell [5] propose using the FOSM method to obtain the mean and standard deviation of loss given $I M$ for individual components, and for computing the covariance in the $L \mid I M$ relationship between different components when computing the standard deviation for the total loss given $I M$. The distribution of the total loss is then combined with the ground motion hazard via numerical integration. Aslani [6] uses the FOSM method only for the computation of the covariance terms when determining the standard deviation in the total loss. That is, Aslani [6] computes the mean and standard deviation of the loss given intensity for a single component are computed using direct numerical integration. For brevity, reference to the approach proposed by Baker and Cornell [5] is denoted as the 'FOSM' approach, while the approach of Aslani [6] is denoted as the 'partial' approach. Solution of the problem via direct numerical integration is denoted the 'direct' approach.

The seismic loss assessment tool, SLAT [15] is used for conducting the loss assessment. In particular, the computational scheme for directly performing the numerical integrations utilizes the magnitude-oriented adaptive quadrature (MAQ) algorithm [16]. The integration parameters used were an error tolerance of $0.005(0.5 \%)$ and a maximum number of function evaluations of 300 (for all computations presented here integral convergence was achieved).

\section{Expected loss given IM}

Earlier in the manuscript, the error associated with the FOSM method for computation of the expected loss given intensity for a single component was illustrated. Three important observations regarding the error in the FOSM approximation were that: (i) the error increased as the dispersion in the $E D P \mid I M$ relationship increased; (ii) the error increased as the 
dispersion in the fragility functions decreased; and (iii) the FOSM method under and over predicted the loss for intensities below and above the intensity causing a $50 \%$ probability of exceedance of the damage state, respectively.

Figure 7a illustrates the computation of the total expected loss given intensity and no collapse $\left(\mu_{L_{T} \mid I M, N C}\right)$ for the entire structure. It is first noted that the partial method yields the same results as the direct method since the expected total loss given no collapse is simply the sum of the expected loss given intensity for each component (Equation 6). Secondly, it is noted that while the FOSM method under predicts the expected loss relative to the direct solution its approximation is surprisingly good relative to the results observed for the approximation of the expected loss given intensity for single components (i.e. Figure 3). The reason for this improved performance for the total loss (compared to the loss for a single component) can be attributed to the third point noted above whereby the negative errors in the estimation of the loss in one component are offset by positive errors in the estimation of loss for another component. Figure $7 \mathrm{~b}$ illustrates the relative error between the FOSM and direct solutions for the total expected loss as a function of $I M$. The two different lines illustrate the error in the expected total loss given no collapse, $\mu_{L_{T} \mid I M, N C}$, and the expected total loss after conditioning on collapse is removed, $\mu_{L_{T} \mid I M}$ [7]. As both the FOSM and direct approaches consider the loss due to global collapse in the same fashion (by combing the collapse fragility curve, $\mathrm{P}_{C \mid I M}$, with the mean loss due to collapse, $\left.\mu_{L_{T} \mid C}\right)$ then as $I M$ increases, and a significant portion of the expected loss is due to collapse (due to an increasing probability of collapse), the relative error will tend to zero as $\mathrm{P}_{C \mid I M}$ approaches unity. 

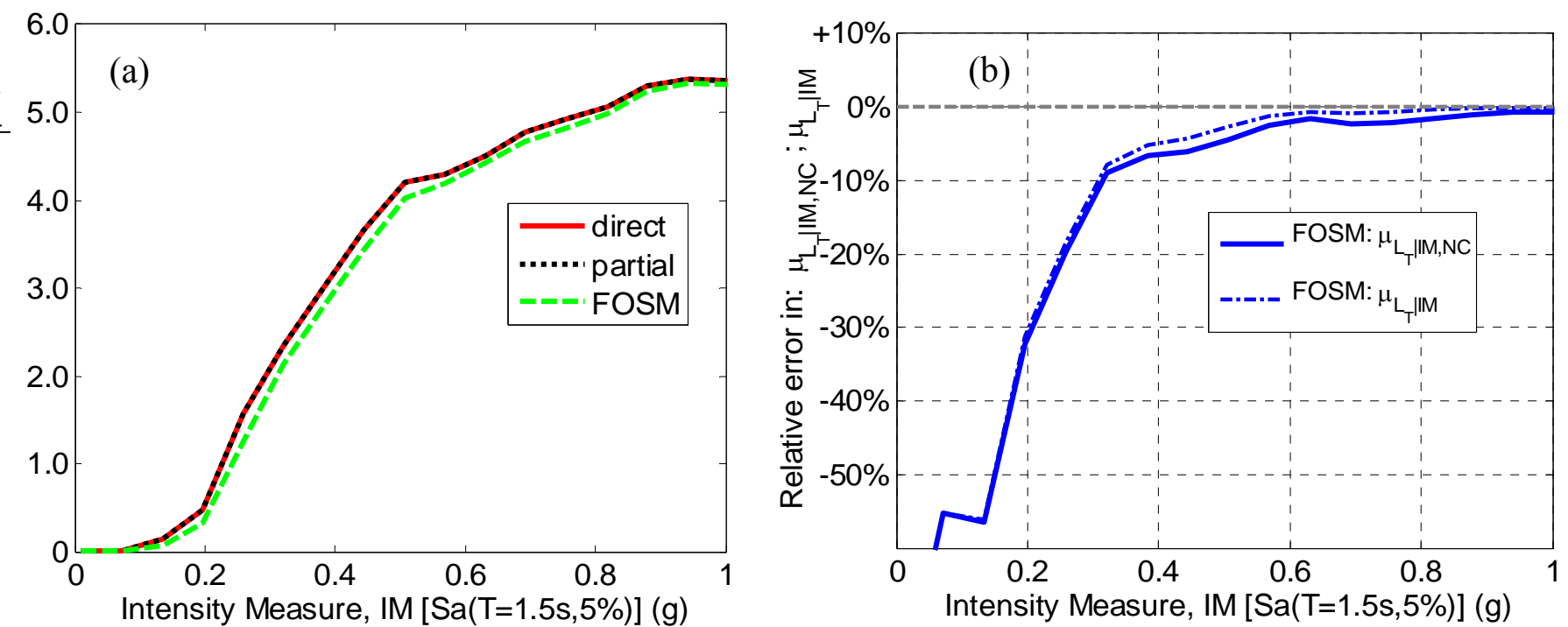

Figure 7: (a) expected total loss given intensity for the case study structure; and (b) relative error of the FOSM approximation as a function of $I M$.

Figure $7 \mathrm{~b}$ illustrates that for $I M>0.3 \mathrm{~g}$ the FOSM approximation results in less than a $10 \%$ under prediction of the expected loss, and the error is below $5 \%$ for $I M>0.5 \mathrm{~g}$ (this corresponds to a ground motion with approximately a $2 \%$ exceedance in 50 years). The fact that the error is quite large for low levels of ground motion is that most components in the structure are subjected to demands which give less than a $50 \%$ probability of damage state exceedance. As it was previously observed that the FOSM method under predicts the loss in individual components for low $I M$ levels, then for these low $I M$ levels, the FOSM method under predicts the expected loss for the majority of components, and therefore the amount of subtractive cancellation is small. When the ground motion hazard curve and the expected loss as a function of intensity, $\mu_{L_{T} \mid I M}$, are convoluted (using direct numerical integration) to obtain the expected annual loss, EAL (i.e. Equation (9)), it was found that the FOSM method gives an under-approximation of the EAL by $15 \%$ compared to that obtained via direct numerical integration.

For this specific structure, over the majority of $I M$ levels, the loss is not dominated by a single type of component. Therefore the aforementioned subtractive cancellation effect means that the FOSM method is relatively accurate for this example. Care should therefore 
be taken on a case-by-case basis that the loss (for a given level of intensity) is not dominated by a single component (or component type). If the loss is dominated by a single component then the accuracy of the FOSM method will likely be reduced, similar to that observed for the parametric study presented earlier for individual components (see Figure 3).

Caution should also be exercised when investigating the deaggregation of loss given $I M$ for use in decision making purposes, because as mentioned above, the contribution of various types of components may be significantly different when using the FOSM method in place of direct numerical integration. Figure 8 illustrates the deaggregation of the expected loss given no collapse for $I M=0.2 \mathrm{~g} S a$ (which has a rate of exceedance of $17 \%$ in 50 years for the site), by fragility type computed using both the direct and FOSM approaches. It is apparent in Figure 8 that while the hierarchy of component contribution to the total loss does not change significantly, there are a few components whose contribution is markedly different between the two solutions. Changes in contributions to the loss of particular note are: server and network equipment reduce from $18 \%$ to $13 \%$; acoustical ceiling reduce from $9 \%$ to $5 \%$; paint increases from $9 \%$ to $15 \%$. These increasing and decreasing proportions can be explained by

(a)

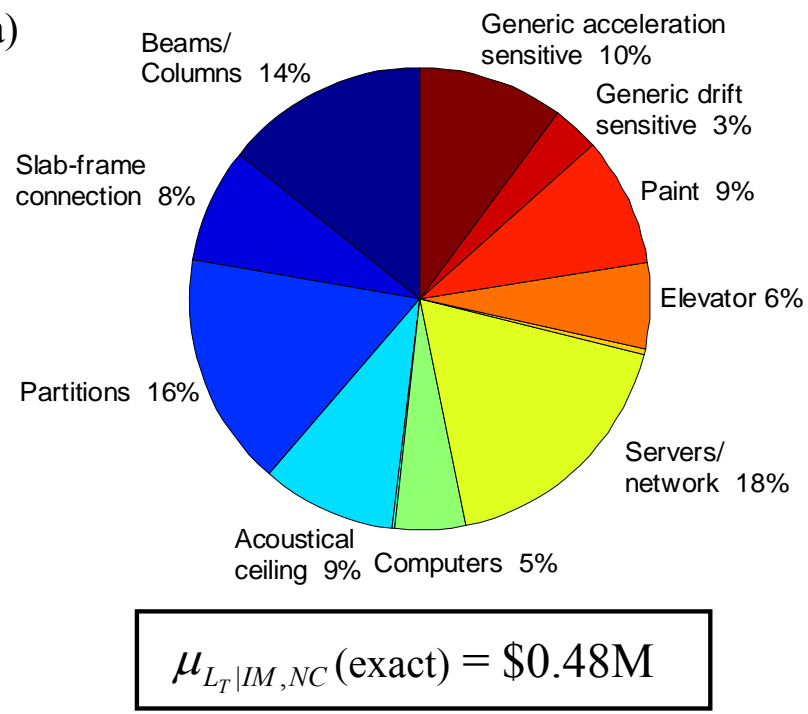

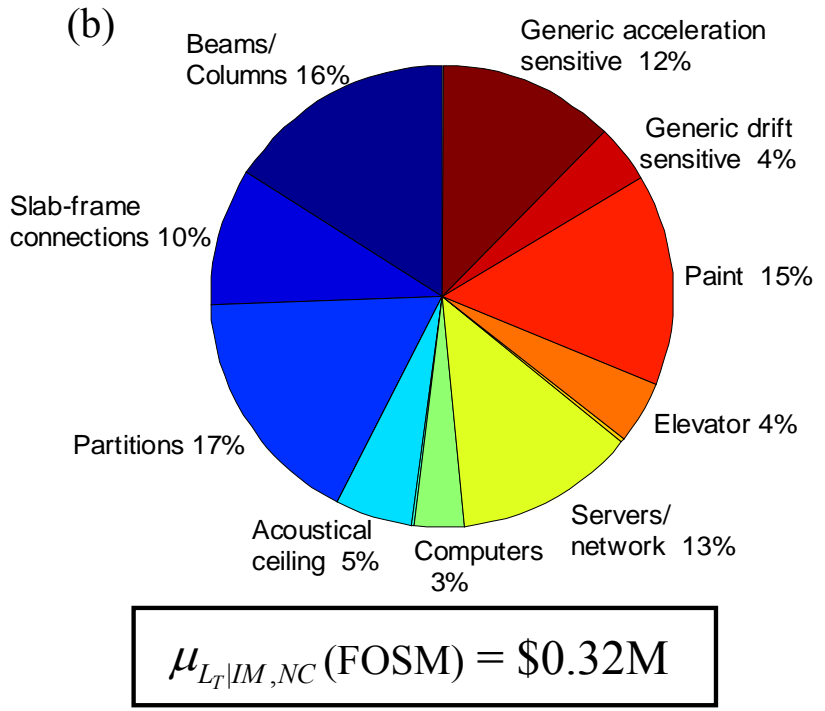

Figure 8: Deaggregation of the expected total loss given no collapse at $I M=0.2 \mathrm{~g} S a$,by fragility type using: (a) direct numerical integration; and (b) FOSM method.

*For the fragility deaggregation components with less than $1 \%$ contribution have not been annotated 
recalling from Figure 4 that the FOSM method under approximates the loss for IM values below that causing the median loss, and over approximates the loss for IM values above the median loss.

\section{Standard deviation in loss given IM}

Figure 9 illustrates the comparison in the computation of the standard deviation in the total loss. As it is beyond the scope of this work to delve deeply into the consideration of correlations in the loss assessment, we will merely consider the upper and lower bounds of perfect and no correlations, respectively. Figure 9a shows the accuracy of the FOSM method for predicting the standard deviation for the no correlation case. Note that since the partial method computes the mean and standard deviation in the loss for individual components using numerical integration, then for the case of no correlations (i.e. the covariance terms in Equation (5) are zero) the standard deviation in the total loss is estimated exactly. Similar to the results observed in the prediction of the standard deviation in the loss for individual components, the standard deviation in the total loss is under predicted by the FOSM method over the entire range of $I M$. Figure 9c illustrates the relative error in the FOSM method for the case of no correlations. In particular, while there is quite a significant difference in the prediction of the standard deviation in the total loss conditioned on no collapse, once conditioning on no collapse is removed (i.e. Equation (8)), the error reduces significantly. This is due to the fact that for the no correlation case the standard deviation in the total loss given no collapse is small relative to the standard deviation in the total loss given collapse (which is computed exactly).

Figure $9 \mathrm{~b}$ illustrates the prediction of the standard deviation in the total loss given no collapse for the case of perfect correlations in the $L|D S, D S| E D P$, and $E D P \mid I M$ relationships. Note that as mentioned previously, perfect correlations in each of the aforementioned three relations does not imply a perfect correlation within the $L \mid I M$ relationship. The error in 
making this assumption is illustrated in Figure 9b, with a relative over-prediction of approximately $20 \%$ (Figure $9 \mathrm{~d}$ ) in the case of no collapse. Figure $9 \mathrm{~b}$ also illustrates the accuracy of the FOSM and partial methods in predicting the standard deviation in the total loss given no collapse. It should be noted that the difference between the two methods is the computation of the variance terms (first part of Equation (5), which the partial method solves exactly, while the FOSM method uses Equation (18)). The computation of the covariance terms (second part of Equation (5)) is approximated using Equation (21) in both FOSM and partial methods. From Figure $9 \mathrm{~b}$ it is apparent that both methods quite significantly under predict the magnitude of the standard deviation.
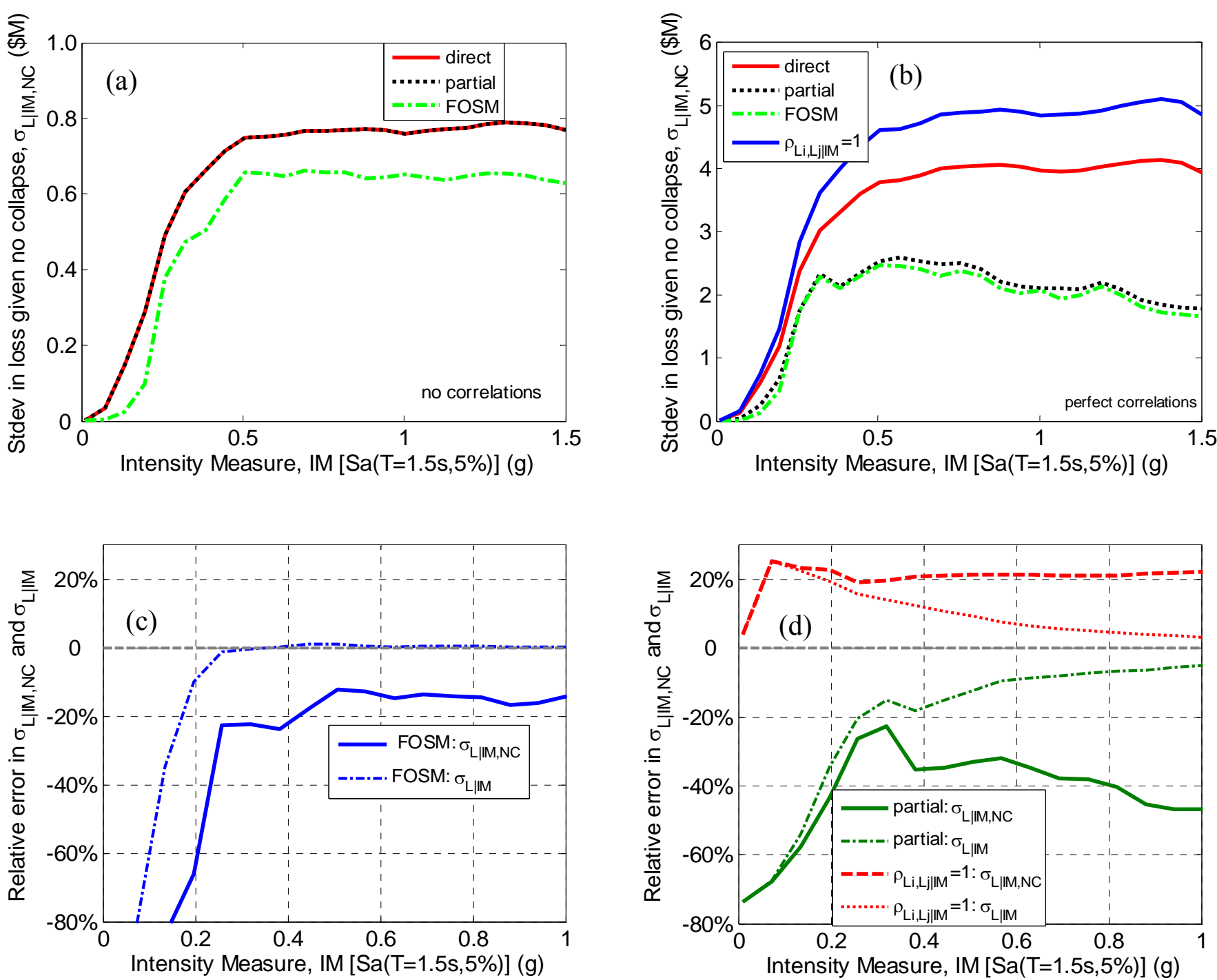

Figure 9: Comparison of the computation of the standard deviation in the total loss: (a) assuming no correlations; (b) assuming perfect correlations; (c) relative error of the FOSM method when assuming 
no correlations; and (d) relative error of FOSM and partial methods when assuming perfect correlations.

Figure $9 \mathrm{~d}$ illustrates that the relative error in the standard deviation using the partial method is always greater than $20 \%$ for the case of no collapse (the error in the FOSM method is not shown, because of its similarity to the partial method). While this error is reduced when collapse is considered, the reduction is not as marked as that of the case of no correlations, with the error greater than $20 \%$ for $I M<0.25 \mathrm{~g}$ and less than $10 \%$ for $I M>0.5 \mathrm{~g}$. This is because the magnitude of the standard deviation given no collapse is about 5 times larger than the case of no correlations (and so the collapse standard deviation does not dominate). Figure $9 \mathrm{~d}$ also illustrates the relative error in assuming that if perfect correlations exist between the $L|D S, D S| E D P$, and $E D P \mid I M$ relationships, then a perfect correlation exists for the $L \mid I M$ relationship. This assumption causes an over-approximation of approximately $20 \%$ in the standard deviation of the loss given no collapse.

\section{Loss hazard}

The loss hazard curve, which gives the annual rate of exceeding various levels of loss, can be obtained from the distribution of the total loss and the ground motion hazard curve, as given by Equation (10). Figure 10a and Figure 10b illustrate the errors in the computation of the loss hazard curve for the two cases of zero and perfect correlations, respectively. For the case of no correlations (Figure 10a), since the error in the standard deviation using the FOSM method (considering both collapse and non collapse cases) is below $10 \%$ once $I M>0.3 \mathrm{~g}$ then the reason for the difference between the direct and FOSM-based loss hazard curves is primarily due to the difference in the computed expected loss given IM (Figure 7). For example, at an exceedance rate of $2 \times 10^{-3}$, values of $\$ 1.3 \mathrm{M}$, and $\$ 1.6 \mathrm{M}$ are obtained for the FOSM and direct methods, respectively. Again it is noted that in the case of no correlations the partial approach is exact and therefore is not shown here. 
Figure $10 \mathrm{~b}$ illustrates the error in the loss hazard curve in the case of perfect correlations. It can be seen that the under prediction of the standard deviation in the total loss by the partial method (and FOSM method) results in an un-conservative loss hazard curve. The conservative assumption of perfect $L \mid I M$ correlations is also shown, which results in a conservative loss hazard curve. For an exceedance rate of $2 \times 10^{-3}$, values of $\$ 0.87 \mathrm{M}, \$ 1.08$ $\mathrm{M}$, and $\$ 1.06 \mathrm{M}$ are obtained for the partial, direct, and perfect $L \mid I M$ correlation cases, respectively. Comparison of Figure 10a and Figure 10b also illustrates the significant effect of uncertainty in the $L \mid I M$ relationship on the shape of the loss hazard curve, and the rate of occurrence of various levels of loss, particularly at low rates of exceedance.
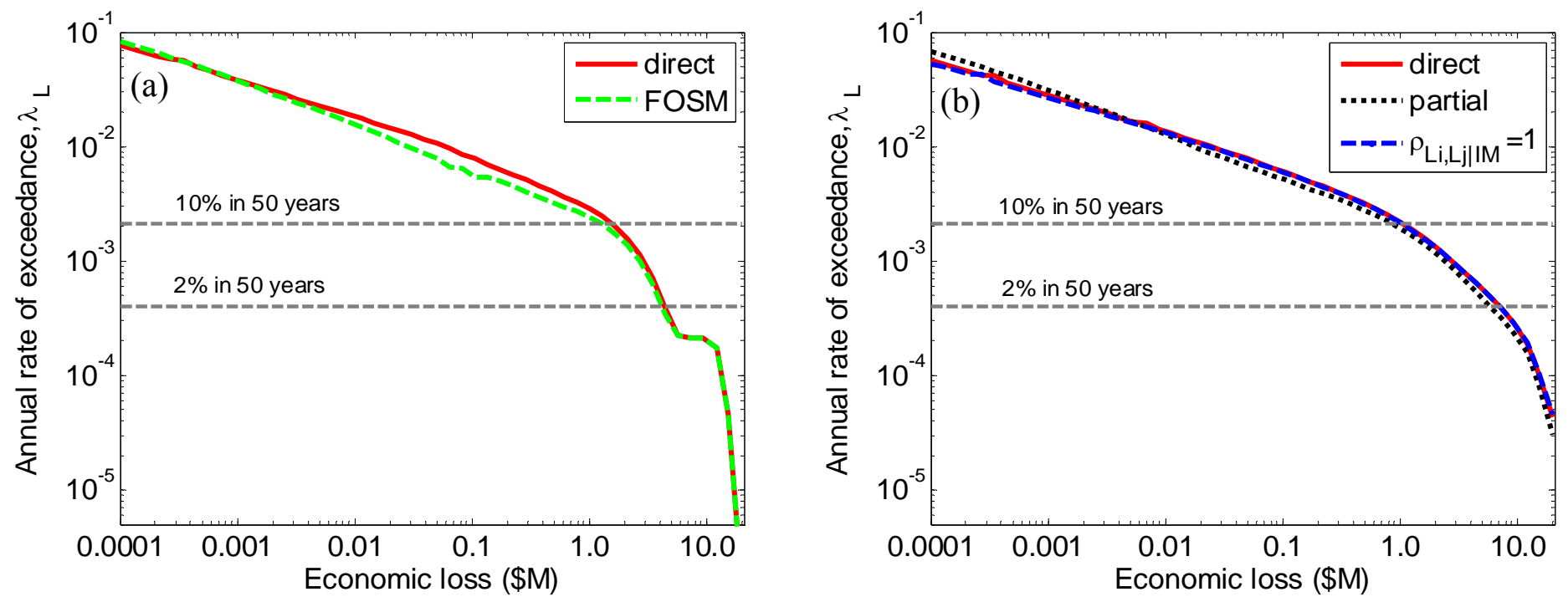

Figure 10: comparison of the computation of the loss hazard curve: (a) assuming no correlations; and (b) assuming perfect correlations.

\section{Computational demand}

The primary benefit with any approximate method is its reduction in effort to the user, whether it be computational as in this case, or expertise required in the input information. Thus, in order to put the accuracy of the aforementioned approximate methods into perspective, it is necessary to focus on their benefits of computational reduction. The case study presented in the previous section represents a good example to illustrate the computational demands in performing a loss assessment, with a total of 115 different 
components monitored over 21 different EDP's.

Table 2 presents the computational times required when in performing the seismic loss assessment on a Pentium 4 processor with $3.0 \mathrm{GHz}$ CPU and $512 \mathrm{MB}$ RAM using the seismic loss assessment tool (SLAT) [17] with 25 output points (i.e. $25 I M$ points for the $L \mid I M$ relations, or 25 Loss points for the loss hazard). The computations use the MAQ integration algorithm [16] with an error tolerance of $0.001(0.1 \%)$. For comparison the computational demand when computing the loss hazard curve with a larger (1\%) error tolerance is also given. Table 2 illustrates primarily two key points, the first being that computation of the loss hazard curve is significantly more demanding than that for the $L \mid I M$ and $E A L$ computations. Secondly, the effect of considering correlations is also significant in increasing the computational demand. The reason for the latter observation is explained in the following sentences. If we consider the computational effort of the mean and standard deviation of loss given $I M$ for a single component to be $2 \bar{n}_{\text {feval }}$, where $\bar{n}_{\text {feval }}$ is the average number of function evaluations required to perform the integration (in Equation (2)), and the coefficient ' 2 ' is for computation of both the mean and standard deviation. Then the total computational work to obtain the mean and standard deviation of the total loss for no correlations is approximately $2 \bar{n}_{\text {feval }} m$, where $m$ is the number of components. When correlations are considered the covariance in the loss given $I M$ between two different components (Equations (6) and (7)) requires approximately $\bar{n}_{\text {feval }}^{2}$ function evaluations, and the second term in Equation (5) indicates that for $m$ components there are $\frac{1}{2} m(m-1)$ covariance computations to be computed. Thus the ratio between the computational effort to evaluate the covariance terms in Equation (5) and the remainder of the terms in Equations (4) and (5) is given by: 


$$
\frac{\operatorname{Comp}\left[\sum \sum \sigma_{L_{i}\left|I M, L_{j}\right| I M}\right]}{\operatorname{Comp}\left[\sum \mu_{L_{i} \mid I M}, \sum \sigma_{L_{i} \mid I M}^{2}\right]} \sim \frac{\frac{1}{2} m(m-1) \bar{n}_{\text {feval }}^{2}}{2 \bar{n}_{\text {feval }} m}=\frac{\bar{n}_{\text {feval }}(m-1)}{4}
$$

Equation (26) illustrates that for the case study structure with $m=115$ components, and given that $\bar{n}_{\text {feval }} \sim 60$ the computational effort to compute the covariance terms is of the order of 1700-times that to compute the other terms in the $L \mid I M$ relation. For the special case of perfect correlations within the $E D P \mid I M$ relationship, the computational demand ratio reduces to approximately $(m-1) / 4$. As Equation (26) illustrates that the computational work to determine the covariance terms is a quadratic relationship of the number of components, $m$, then it is noted that the use of deaggregation to determine if any components offer insignificant contribution to the loss estimation can significantly reduce the computational demand. For the case study presented in the previous section, up to 15 components could have been removed with a less that $2 \%$ effect on the results, which would reduce the computational demand by approximately $38 \%$.

Table 2: Computational times (in seconds) for performing seismic loss estimation

\begin{tabular}{|c|c|c|c|c|c|c|}
\hline & \multicolumn{3}{|c|}{ No correlations } & \multicolumn{3}{c|}{ Perfect correlations } \\
\hline relationship & FOSM & Partial & Direct & FOSM & Partial & direct \\
\hline $\begin{array}{c}\mu_{L_{T} \mid M}, \sigma_{L_{T} \mid M}^{2} \\
\text { Eq. 6-10) }\end{array}$ & 0.64 & 1.67 & 1.67 & 4.8 & 5.8 & 78.2 \\
\hline$\mu_{L_{T}}($ Eq 11) & 2.47 & $15.0 \mathrm{~s}$ & 15.0 & 43.8 & 53.1 & 705 \\
\hline$\lambda_{L_{T}}(\mathrm{Eq} \mathrm{12)}$ & $11.2(3.97)^{\star}$ & $103(18.3)^{\star}$ & $103(18.3)^{\star}$ & $391(119)^{\star}$ & $480(142)^{\star}$ & $6200(1205)^{\star}$ \\
\hline
\end{tabular}

${ }^{*}$ Computation times using an error tolerance of $1 \%$ in brackets.

\section{CONCLUSIONS}

The efficacy of approximate methods of uncertainty propagation in seismic loss estimation has been investigated in this manuscript. The approximate methods are based on the use of the first-order second-moment (FOSM) method which uses a first-order Taylor Series approximation to compute the first two moments of functions of random variables. The 
FOSM method was used primarily to determine the moments of the loss given intensity measure $(L \mid I M)$ relationship. It was illustrated that the accuracy of the FOSM method is directly related to the uncertainty in the $E D P \mid I M$ relationship, with increasing error as the uncertainty increases. As the error in the approximation of the mean loss given intensity for a given component can be either an over or under approximation, then when computing the mean loss given intensity for the entire structure, some cancellation errors occur, with relative errors up to $50 \%$ for small $I M$ values and reducing error with increasing $I M$ as more components contribute to the total loss. The same cannot be said for the approximation of the standard deviation of the loss given intensity, which was almost always under predicted by more than $20 \%$ for the case-study structure considered. Despite the cancellation errors giving a small total error in the mean loss given intensity for the entire structure, care should be taken in deaggregation of the results, which tend to have significantly larger errors compared to the exact solution. The effects of the errors in the $L \mid I M$ relationship on the resulting loss hazard curve were also investigated. Computational times to conduct the analysis on a standard PC indicate that the loss hazard computation was approximately 8 - and 80-times more computationally demanding than computation of the $L \mid I M$ and expected annual loss $(E A L)$ decision variables, and that consideration of component correlations is also demanding. Both approximate and exact methods have their pros and cons in various situations. This paper therefore elucidates the situations in which an analyst may lean toward one of the methods over the other, be it based on computational demands or accuracy tolerance.

\section{ACKNOWLEDGEMENTS}

Financial support of the first author by the New Zealand Tertiary Education Commission is greatly appreciated. Discussions between Dr. Jack Baker and the first author are gratefully acknowledged. 


\section{REFERENCES:}

[1] Cornell C. A. and Krawinkler H. Progress and challenges in seismic performance assessment, PEER Center News, vol. 3, 2000.

[2] Deierlein G. G., Krawinkler H., and Cornell C. A. A framework for performancebased earthquake engineering, in 7th Pacific Conference on Earthquake Engineering, Christchurch, New Zealand, 2003.

[3] Cornell C. A. A probability based structural code, Journal of the American Concrete Institute, vol. 66, pp. 974-985, 1969.

[4] Baker J. W. and Cornell C. A. Uncertainty Specification and Propagation for Loss Estimation using FOSM Method, Peer Report No 2003/07 2003.

[5] Baker J. W. and Cornell C. A. Uncertainty propagation in probabilistic seismic loss estimation, Structural Safety, vol. 30, pp. 236-252, 2008.

[6] Aslani H., Probabilistic earthquake loss estimation and loss disaggregation in buildings, Ph.D. Thesis, John A. Blume Earthquake Engineering Centre, Dept. of Civil and Environmental Engineering Stanford University, Stanford, CA, 2005, 382.

[7] Bradley B. A., Dhakal R. P., Cubrinovski M., MacRae G. A., and Lee D. S. Seismic loss estimation for efficient decision making, Bulletin of the New Zealand Society for Earthquake Engineering, vol. 42, pp. 96-110, 2009.

[8] Ang A. H. S. and Tang W. H. Probability Concepts in Engineering Planning and Design vol. Volume I - Basic Principles: John Wiley \& Sons, Inc., 1975.

[9] Melchers R. E. Structural Reliability Analysis and Prediction. Chichester: John Wiley and Sons, 1999.

[10] Mitrani-Reiser J., An Ounce of Prevention: Probabilistic Loss Estimation for Performance-based Earthquake Engineering, Ph.D. Thesis, California Institute of technology, Pasadena, CA, 2007, 173.

[11] Zareian F. and Krawinkler H. Assessment of probability of collapse and design for collapse safety, Earthquake Engineering and Structural Dynamics, vol. 36, pp. 19011914, 2007.

[12] Haselton C. B., Assessing Collapse Safety of Modern Reinforced Concrete Moment Frame Buidlings., Ph.D. Thesis, Department of Civil and Environmental Engineering Stanford University, Stanford, CA, 2007, 312.

[13] Bull D. K. and Brunsdon D. Examples of Concrete Structural Design to New Zealand Standards 3101, New Zealand 1998.

[14] Standards New Zealand. NZS 3101 1995: Part 1: Concrete Structures Standard, Wellington, NZ, 1995.

[15] Bradley B. A. User manual for SLAT: Seismic Loss Assessment Tool version 1.14, Department of Civil and Natural Resources Engineering, University of Canterbury, Christchurch, New Zealand, University of Canterbury Research Report No.2009-01, 2009.

[16] Bradley B. A., Lee D. S., Broughton R., and Price C. Efficient Evaluation of Performance-based Earthquake Engineering Equations, Structural Safety vol. 31, pp. 65-74, 2009. 
[17] Bradley B. A. SLAT: Seismic Loss Assessment Tool, version 1.12 user manual, Department of Civil Engineering, University of Canterbury, Computer Program Library, Christchurch, New Zealand 2008. 